\title{
Affective psychotherapy in post-traumatic reactions guided by affective neuroscience: memory reconsolidation and play
}

REVIEW

\author{
Göran Högberg',2 \\ Davide $\mathrm{Nardo}^{3}$ \\ Tore Hällström ${ }^{4,5}$ \\ Marco Pagani ${ }^{6,7}$ \\ 'Department of Women's and \\ Children's Health, Child and \\ Adolescent Psychiatric Unit, Astrid \\ Lindgren Children's Hospital, \\ Karolinska Institutet, Stockholm, \\ Sweden; ${ }^{2}$ BUP Huddinge Child and \\ Adolescent Psychiatry, Stockholm, \\ Sweden; ${ }^{3}$ Neuroimaging Laboratory, \\ Santa Lucia Foundation, Rome, \\ Italy; ${ }^{4}$ Department of Clinical \\ Neuroscience, Section for \\ Psychiatry/Huddinge, Karolinska \\ Institutet, Stockholm, Sweden; \\ ${ }^{5}$ Department of Neuroscience, \\ Section of Psychiatry and \\ Neurochemistry, Unit for \\ Neuropsychiatric Epidemiology, The \\ Sahlgrenska Academy, University of \\ Gothenburg, Gothenburg, Sweden; \\ ${ }^{6}$ Department of Nuclear Medicine, \\ Karolinska Hospital, Stockholm, \\ Sweden; ${ }^{7}$ Institute of Cognitive \\ Sciences and Technologies, CNR, \\ Rome, Italy
}

Correspondence: Göran Högberg BUP Huddinge, Paradistorg 4, I4I 47 Huddinge, Sweden

Tel +4685I 452900

Fax +46851452905

Email gor.hogberg@gmail.com
This article was published in the following Dove Press journal:

Psychology Research and Behavior Management

4 July 2011

Number of times this article has been viewed

\begin{abstract}
This paper reviews the affective neuroscience dealing with the effects of traumatic events. We give an overview of the normal fear reactions, the pathological fear reaction, and the character of emotional episodic memories. We find that both emotions and emotional memories are a tripartite unit of sensory information, autonomic reaction, and motor impulse (the PRM complex). We propose that emotions and movements are part and parcel of the same complex. This is our main finding from the review of affective neuroscience, and from here we focus on psychotherapy with post-trauma reactions. The finding of the process of memory reconsolidation opens up a new treatment approach: affective psychotherapy focused on reconsolidation. The meaning of reconsolidation is that an emotional memory, when retrieved and being active, will rest in a labile form, amenable to change, for a brief period of time, until it reconsolidates in the memory. This leads us to the conclusion that emotions, affects, must be evoked during the treatment session and that positive emotion must come first, because safety must be part of the new memories. In the proposed protocol of affective psychotherapy based on reconsolidation the emotional episodic memory is relived in a safe and positive setting, focused in turn on the sensory experience, the autonomic reaction, and the motor impulse. Then it is followed by a fantasy of a different positive version of the same event. All in all treatment should provide a series of new memories without fear related to the original event. With the focus on the motor program, and the actions, there is a natural link to art therapy and to the mode of play, which can rehearse and fantasize new positive actions.
\end{abstract}

Keywords: post-traumatic, PTSD, affective neuroscience, memory reconsolidation, psychotherapy, play

\section{Aim}

In this article the aim is to create an outline of the findings from neurocognitive science of dysfunctional reactions in the aftermath of traumatic experiences. From this contour we suggest a protocol of treatment principles.

\section{Trauma across diagnoses - trauma spectrum disorders}

Over the years many trauma-related diagnoses have emerged in psychiatry, several of of which were related to extreme experiences such as war and termed shellshock, war neurosis, traumatic neurosis, and disordered action of the heart. Oppenheim's term, from 1892, traumatic neurosis, opens the descriptions even for traumas of civilian origin. ${ }^{1}$ With the advent of the DSM-III in 1980, epidemiological studies were enabled by a common definition of a post-traumatic stress disorder (PTSD). 
The National Comorbidity Survey in the United States reported a lifetime prevalence rate of $10.4 \%$ in women and $5 \%$ in men. ${ }^{2}$ The lifetime prevalence of PTSD in Sweden is estimated to be $7.4 \%$ for women and $3.6 \%$ for men. ${ }^{3}$

Trauma reactions are an important part of the general outpatient psychiatry. In a survey of an outpatient clinic, conducted in a part of Stockholm in which live many immigrants, $77 \%$ of patients had been exposed to several traumas. The prevalence of PTSD was estimated to be $69 \%$ within the Iranian, 59\% within the Arabic, 53\% within the Turkish, and $29 \%$ within the Swedish patient cohorts. ${ }^{4}$

Traumatic experiences followed by negative emotional memories are related to depression: in a meta-analysis with 14,240 subjects there was a strong correlation between childhood adverse experiences and depression. ${ }^{5}$ The symptoms of patients with severe difficulties of mood regulation and with increased suicidal tendencies have been described to result from previous traumas such as rape and abuse. ${ }^{6}$

Trauma tends also to be a background factor in psychotic manifestations such as hallucinations. ${ }^{7}$ The inner experience of the psychotic episode as well as the encounter with the health-care and law-enforcement systems during the psychotic episode may itself be a traumatic event with post-traumatic sequelae. ${ }^{8}$ A factor contributing to psychotic symptoms is strong anxiety, and progressive relaxation was useful for dampening these symptoms. ${ }^{9}$ Another clinically important reaction is trauma-related dissociative psychosis, which can be characterized as a long permanent flashback, the patient reliving the traumatic preceding event in a symbolic disguise. ${ }^{10}$

The Adverse Childhood Experiences (ACE) study including 17,337 subjects disclosed a strong relationship between adverse childhood experiences and depression, suicide attempts, alcoholism, drug abuse, and other negative health outcomes. ${ }^{11}$ Traumatic experiences in childhood with longer duration - as in cases with trauma and neglect - impair self-regulation function such as mood regulation and constancy in relations. This is described in new diagnoses such as "complex PTSD" and “developmental trauma disorder". ${ }^{22}$

The wide array of psychopathology related to traumatic experiences and fear reactions that go under the heading of trauma spectrum disorders provide the common ground for many of the concomitant diagnoses in severe post-trauma reactions.

\section{Affective neuroscience and psychotherapeutic options}

The study of emotions is an expanding field in neurocognitive science. Both clinical and preclinical studies shed light on the networks in the brain that are involved in trauma reactions. Rodent models of stress, mimicking human fear reactions, have been proposed. With humans, technologies capable of registering brain activity are rapidly being developed. Electrical activity of the brain's cortex can be recorded using electroencephalography (EEG). Single photon emission computerized tomography (SPECT), positron emission tomography (PET), and functional magnetic resonance imaging (fMRI) are techniques capable of detecting relative changes in electric activity/blood flow/oxygenation, respectively, which are indicative of differences in regional brain activity. ${ }^{13}$ The purpose of this paper is to extract the main findings relating to emotional memory and effects of treatment, and to tentatively suggest psychotherapeutic options, following findings of affective neuroscience.

\section{Fear reactions in episodic memory}

Fearful experiences are not automatically followed by a subsequent pathological fear reaction. Normally, a traumatic event is followed by an acute stress reaction that naturally subsides within a month. The organism learns a no-fear signal; the danger is over and the fear reaction is no longer functional. ${ }^{14}$ When a pathological post-traumatic reaction develops, the fear reaction is preserved and the organism establishes a state of alarm and action-preparedness. This heightened fear reactivity can manifest itself when a person is quiet and trying to relax, for instance when going to bed and during sleep, having gained an automatic quality of being a default state. Sensory experiences associated with the original traumatic situation can trigger the fear reaction into more explicit states of pathological fear such as anxiety, panic, unrest, and destructive actions. At the bottom lies the original traumatic situation, but what then follows has to do with the way the organism is processing that experience, that is, it has to do with memory. Memory is a popular concept with many aspects. The memory of the past experiences has many qualifying terms, such as declarative, narrative, explicit, and personal memory, but here we choose the broader concept of episodic memory since this can also include sensory and hidden elements that are not declarative or explicit, but nevertheless part of the trauma episode.

\section{Emotion and memory}

The organism is set to act in relation to the environment; thus emotions can be conceptualized as guiding mental states to implement appropriate motor programs. Emotions are like a coloring of the memory of an event, basically divided into positive or negative. This division has been 
described as two distinct reaction patterns. ${ }^{15}$ With positive emotions there is an appetite and approach behavior implicated. With negative emotions - such as fear and anger - an active escape from danger, withdrawal, or withdrawal after attack occurs. This is in accordance with a multidimensional definition of feelings by Osgood et al ${ }^{16}$ involving valence, intensity, and level of control. The episodic memory activates networks with reciprocal pathways linking medial temporal lobe, prefrontal cortex, thalamus, hypothalamus, brainstem, striatum, anterior cingulated, sensorimotor areas, and cerebellum. In the ensuing text we give examples thereof.

When positive emotions are evoked by a script, there is an increased activity in the anterior cingulated, anterior temporal cortex as well as the ventral globus pallidus, indicating autonomous arousal as well as action preparation. ${ }^{17}$ The viewing of both negative and positive movies, as well as recall-generated emotions, were shown to be associated with increased activity in the medial prefrontal cortex and thalamus. ${ }^{18}$ The early visual induction of feelings activates somatosensory systems. ${ }^{19}$ Sabatinelli et a ${ }^{20}$ found that both negative and positive emotions evoked by scripts lead to increased activation in areas involved with preparatory motor action.

It seems that memory is not located in a certain area of the brain but is rather a temporal coordination of the reactivation of sensory, autonomic-related, and motor areas that were activated at the event of imprinting. To quote Damasio "This proposal rejects a single anatomical site for the integration of memory and motor processes and a single store for the meaning of entities of events. Meaning is reached by time-locked multiregional retroactivation of widespread fragment records". ${ }^{21}$

Estimations of the future is also a part of the memory function, being memories of fantasies and calculations done in order to have understanding and control of what may be arriving ahead in time.

\section{The episodic memory-perception, reaction, and motor impulses (a PRM-complex)}

The definition of memory by Domjan and Burkhard, ${ }^{22}$ "A theoretical term used to determine instances in which a subject's current behavior is determined by some aspects of his previous experience", leads towards the point of memory and action. The psychotherapy researcher Wolpe ${ }^{23}$ argued that an emotional picture could be seen as a neural event that partly replicates the previous event itself in its aspects of sensations and impulses. Lang ${ }^{24}$ developed a theory pertaining to the emotional picture where he describes the emotional image as an action sequence with a series of propositions of sensory experiences and action alternatives. Thus, there also is an activation of motor impulses. Both the premotor cerebral cortex and the cerebellum are activated in imagined movement. ${ }^{25}$ The cerebellum engages in the reassignment of motor response. ${ }^{26}$ The autonomic regulation and its body representation in emotions are associated with the anterior cingulate and the insula. ${ }^{27,28} \mathrm{We}$ can conceive episodic memory as an organized tripartite complex with perception, reaction, and motor impulse (the PRM-complex).

Consciousness is a balance between situational input and internal input with remnants from previous experiences. The thalamus involves systems for sensory information and sensorimotor integration and can be activated by external, as well as by internal stimuli. ${ }^{29}$ The episodic memory is not a slide show with one distinct episode presented at a time but rather like the northern lights, ever waxing and waning associative networks, some aspects being in the front, soon to be replaced by another set of combinations..$^{30}$ Considering this, it has also to be understood that episodic memories are always having at least some taint of emotion. Moreover, episodic memory can present itself in parts, showing fragments in various sensory and autonomic modalities. This means that episodic memory might appear as an inner vision, a sound, or just a hint - a brief sensation in the belly or a strong pain in the chest.

\section{The hemispheric lateralization of emotional memories}

A consistent and intriguing finding in affective neuroscience has been the lateralization of emotions. This field started with the findings by Jules Bernard Luys ${ }^{31}$ of emotional responses in hemiplegic patients, left hemiplegic patients being more emotional and anxious and right hemiplegic patients being more inactive and passive.

With negative feelings there seems to be a shift of activity in the brain predominately towards the right hemisphere. ${ }^{15}$ In our study on trauma-exposed train drivers, upon trauma memory, a relative increase of activity in the right hemisphere was noted with SPECT. ${ }^{32}$ With procaine-induced mental states negative emotions correlated positively with left amygdala activation whereas positive emotions correlated negatively with left-sided amygdala activation. ${ }^{33}$

In 24 women with PTSD, Metzger et $\mathrm{al}^{34}$ found that right-sided parietal EEG activation during resting state was associated with PTSD arousal symptoms. 
Achuff $^{35}$ notes in a review that there are two theories. The right-hemisphere model proposes that the right hemisphere is dominant over the left hemisphere for all forms of emotional expression and perception. The valence model suggests that the left hemisphere is dominant for positive emotion (approach-appetite) and the right hemisphere for negative emotion (withdrawal-avoidance). There are good arguments to both models, and a vast literature is summarized in Alves et al. ${ }^{36}$

However, there might also be an individual difference in hemispheric activation in emotional arousal. Schiffer et $\mathrm{al}^{37}$ found a lateral shift to the right hemisphere in about $70 \%$ of subjects who had been maltreated as children. In the other $30 \%$ the shift was towards the left. The technique used in that study was EEG during a neutral or upsetting memory. Those authors concluded that there is a lateralization of emotional memory that can be either right- or left-sided, such as other lateralization qualities, for instance left- or right-handedness. We will return to this subject discussing the importance of the lateralization of emotions for the treatment suggested below.

\section{The sympathetic and parasympathetic systems in fear memory}

In the autonomic nervous system the sympathetic system is engaged in energy mobilization and gross motor activity while the parasympathetic system is associated with restorative and vegetative functions. These two branches are in constant dynamic interaction as described by Thayer and Brosschot ${ }^{38}$ in 2005. It is suggested that the default reaction is the sympathetic activation to novel stimuli, thus favoring safety activity such as the fight or flight response. Thus, there is a dis-inhibition of the parasympathetic system in the fear reaction, the tonic inhibition being restored afterwards. Such inhibition is dependent on the prefrontal cortex, forming a top-down regulation. When the prefrontal cortex activity is lowered there is a withdrawal of parasympathetic inhibition, and this leads to an increased sympathetic dominance, associated with defensive circuits. Heinzel et $\mathrm{al}^{39}$ showed emotional pictures to a sample of healthy subjects during functional magnetic resonance imaging, and found a decreased signal in orbitomedial prefrontal cortex. This suggests a decrease of the frontal tonic inhibition in visually elicited fear reaction. The brainstem-midbrain is implicated in the regulation of autonomic response by nuclei as for instance locus coeruleus (noradrenergic). The amygdala, which evaluates the positive or negative valence of external and internal stimuli, activates the fear system upon perceived danger. The hippocampus, as well as the amygdale, attaches the fear reaction to new situations by associative learning, reminding of the original danger. ${ }^{40}$

Kosten et $\mathrm{al}^{41}$ found increased 24-hour urine excretion of adrenaline and even more pronounced increase of noradrenaline in nine Vietnam War veterans. Breslau ${ }^{42}$ reported from an epidemiological sample that catecholamines (noradrenaline, adrenaline, and dopamine) were elevated in PTSD-subjects compared with controls without PTSD. Night secretion of catecholamines was reported to be elevated in PTSD subjects. ${ }^{43}$ However, Murburg et $\mathrm{al}^{44}$ found that basal sympathetic nervous system functioning was not increased in PTSD patients, and suggested that the increases of catecholamines found indicated increased sympathetic reactivity. Thus PTSD might be defined as an anxiety disorder with a dysregulated balance between inhibitory and excitatory impulses at trauma-trigger-related exposure. O'Donnell et al reviewed the topic of the noradrenergic neural networks and symptoms of PTSD. ${ }^{45}$ The reasoning in this paragraph illustrates the need to activate both the parasympathetic and the sympathetic systems during therapy.

\section{Pathological fear memory - the flashback}

Pathological fear reactions are fear memories that are lacking the safety signal in a situation that is not dangerous. ${ }^{46}$ Reaction activates perceptions, autonomous reaction, and impulse to act. This discord between environment, perception, and action severely hampers the smooth regulation of mood, which is necessary for functional interaction with the environment. A central concept in pathological fear reactions is the flashback.

The term flashback originates from drug experiences in which drug users reported spontaneous and fearful reexperiencing of elements of the drug experience. This hallucinatory experience can be present in different sensory modalities such as auditory and visual hallucinations. In a study by Yui et $\mathrm{al}^{47}$ of spontaneous recurrences to amphetamine psychosis, there were higher plasma norepinephrine levels during the flashbacks. In a study of brain activity during flashback after script-driven symptom provocation in PTSD subjects, Osuch et $\mathrm{al}^{48}$ used script-driven symptom provocation in patients with longstanding PTSD to elicit a fear reaction that was studied with PET measuring cerebral blood flow. They found a direct correlation between flashback intensity and activity in the brainstem, areas associated with motor control, complex visual/spatial cues, and memory. There was an inverse correlation with prefrontal areas.

There are also findings in two studies of an altered cortico-thalamic activity during the flashback experience. ${ }^{49,50}$ 
These changes in the cortico-thalamic loop suggest a focus on internal perception during intense fear memory, the thalamus being also a sensory gating area.

The fear memory with "de novo" quality can be presented in several sensory modalities, but also fragmented or in only one modality. This suggests that there could be only a sound, a fragment of a scene, or a strong autonomous reaction, and this is the way trauma memories might appear in clinical states such as panic anxiety.

In summary, the key phenomenon of trauma-reaction pathology - the memory disturbance with reliving episodic memory - seems to be defined by an increased sympathetic tone, a disinhibition of frontal control, a turning to internal sensory perception, and an activation of cerebral networks reminiscent of conditions at encoding. Such re-experiencing memory can be as brief as short flashbacks but also longer and more debilitating with prolonged flashbacks in intensive anxiety states; it can even be perceived to last for days in cases with trauma-related dissociative psychosis.

As far as we are aware there is no knowledge of the brain being organized across our diagnostic categories in trauma reactions, but rather that it works with continuously balancing feedback systems in order to achieve adaptation to the environment.

We conclude that there is a physiology of the traumatic reliving memory that is important in treatment.

\section{Changes following psychotherapy}

In a review of functional neuroimaging, Linden ${ }^{51}$ concluded from four studies with phobia and panic disorder that the most consistent finding following successful cognitive behavioral treatments was decreased activity in the limbic and paralimbic areas. A study on eye movement desentization and reprocessing (EMDR) therapy was done by Pagani et $\mathrm{al}^{52}$ with traumatized subjects from occupational hazards. The results were that in post-treatment there was a normalization of a previous higher activity in medial temporal lobe, fusiform gyrus, and primary visual cortex. Bryant et $\mathrm{al}^{53}$ showed that poor response to psychotherapy was associated with greater bilateral amygdala and anterior cingulate activation in response to fearful faces. These findings indicate a change in frontalmedial temporal lobe balance, implying that frontal control of emotions is part of the beneficial treatment effect.

\section{Reconsolidation of emotional memory}

In a seminal study by Nader et $\mathrm{al}^{54}$ it was described how a consolidated memory became susceptible to change, either by reinforcement or attenuation, during a short time after retrieval. A similar window of memory change (reconsolidation) was shown in humans with declarative memory by Forcato et al. ${ }^{55}$ The importance of the theory of memory reconsolidation is that it supports memory transformation as a psychotherapeutic strategy. This concept means that the original memory is not constantly remembered in its initial form, but consists of layer upon layer of modifying new experiences. When trauma reactions develop into chronic states such as severe PTSD the new memories are fear memories, thus deepening and spreading the fear reaction, but with natural attenuation the reverse is true.

Nadel and Moscovitch ${ }^{56}$ argue that the hippocampus is activated in episodic memory retrieval leading to a re-encoding. This means that reactivated memories are made stronger while others fall into oblivion, and that several short reconsolidations are more efficient than one prolonged consolidation in creating stable episodic memories. ${ }^{57}$

Monfils et $\mathrm{al}^{58}$ conducted an experiment with rats where there was an extinction training in the time window of reconsolidation after presentation of a conditioned stimulus. Results showed that the conditioned fear response did not reappear upon provocation, and the authors suggest that a new - alternative or combined - memory trace was formed. This memory trace was not associated with a fear reaction.

For human learning, Forcato et $\mathrm{al}^{59}$ showed that a consolidated memory recalled by a reminder is open to the addition of new information during a short time span until reconsolidation takes place.

\section{The motor reaction in fear reactions}

In rodents, the central nucleus of the amygdala projects to the brainstem nuclei and to the hypothalamus, which is related to passive expression of fear. In contrast, the basal nucleus of the amygdala projects to the striatum, which is involved in active anxiety-diminishing motor coping strategies. $^{60}$

Motor response is a central part of emotional memory, and we hypothesize that motor planning and motor impulses underway - but not being consummated - are a part of the pathological fear response that might be related to different aspects of pathology such as pain, restlessness, escape impulses, and aggressive and self-aggressive impulses. In Panksepp's ${ }^{61}$ dual-aspect monism approach, he underscores 
the emotional action systems as being both experience and action. Nardo et $\mathrm{al}^{62}$ showed that with PTSD following occupational trauma there was a higher activity in the insular cortex as well as in the basal ganglia, suggesting both increased awareness of autonomic arousal and increased motor activation.

It is well known that a trauma in which the victim is immobilized is often related to severe post-trauma symptomatology. Could it be that immobilization leads to the passive expression of fear with a high level of anxiety, and that active coping motor activity during the traumatic event leads to subsequent less severe later manifestations of fear reactions?

In this paragraph we have underlined that there is a motor component to emotions, and we suggest that the motor activity inherent in the emotional response is related to symptoms, and must be addressed during treatment.

\section{A manualized psychotherapy protocol}

From the emerging neurocognitive studies of emotion we propose a set of treatment principles to form a manualized protocol for affective psychotherapy. The exact techniques may vary, leaving space for different theoretical and practical orientations. The basis of this protocol are the premises that: 1) episodic memory is also emotional; 2) emotions are a tripartite unity of perception, autonomic reaction, and motor impulses (the PRM-complex); and 3 ) valence, intensity, and control are key aspects of emotions to be treated.

\section{The aim of the treatment}

The aim of the treatment in cases of dysfunctional fearmemory reaction is to change the valence and the intensity of the traumatic memory and to increase control of memory retrieval. The goal of the treatment is to change a reliving intruding memory into a more distant episodic memory.

\section{The mechanism of the treatment}

The proposed way to change the traumatic memory is by reconsolidation. The emotional memory must be accessed and re-experienced: it is then in a labile state that is amenable to change with for instance imaginal exposure, focusing on the three aspects of the emotion (the PRM-complex). Afterwards, it can be reconsolidated as a new memory that does not produce fear reactions. It can also be supplemented with a positive fantasy about the initial event, thus creating associative links to alternative affective experiences.

\section{The means of the treatment}

\section{The positive and negative emotion must} be activated

Both the negative and the positive emotion must be activated during the treatment session. Activating only negative emotions is counterproductive, because it may lead to a symptom rehearsal that will be consolidated in the memory system as a new negative fear reaction. Thus, it is imperative to start with the positive emotions. The need to activate both negative and positive affects can be associated with the lateralization of emotions in the brain. We suggest that instructions that activate both hemispheres of the brain help in arousing both negative and positive emotions. Activities engaging sensory stimulation such as movement, drawing, listening to music, specifically looking at something, or following a moving object with the eyes are such examples. Presumably they are active to both brain hemispheres.

At first, positive emotions must be accessed. This is needed because positive affect must be part of the new memory to be produced during the session. There should be no activation of negative affect in case positive affect cannot be evoked. In such a case, a longer time and more sessions must be spent to reach a positive affect. Activating positive affect means increasing the parasympathetic tone. This can be achieved by relaxation exercises: breathing and meditation, safe-place imagery, perhaps walking on a treadmill or bicycling on a spinning cycle. Also other aspects of safety in the treatment setting are of importance, such as stage of change process and preferences matching. ${ }^{63}$ Once activated, the positive emotion can be anchored in a scene or image with focus on all senses. This means that specific instruction can be given to note the sight, hearing, proprioception, gustatory feeling, and total body sensation in a state of safety and control. After this, instructions are given to connect this positive sensory state with mental clues of retrieval.

\section{The emotional memory as a PRM-complex to be changed}

The emotional memory is composed of sensory, autonomic, and motor activations. In severe fear reactions, as seen in flashbacks occurring in PTSD, there is a more pronounced activation of motor areas in the brain. The emotional memory is not seen as a depiction of an earlier event, but as a blueprint for action, a script, a planned scene. This could be conceived as a PRM-complex. With brief instructions, such as taking a deep breath, the patient can be focused on the 
inner experience of the negative emotional memory. This is similar to Elman ${ }^{64}$ hypnotherapy with short inductions into a state of focus on inner sensations. The proposed protocol is then to: A) re-experience the event in vitro, ie, by mental imagery, an inner film with focus on the sensory experiences; B) experience a version with focus on autonomous reactions; and $C$ ) experience a version with imaginal acting out of the motor impulses, the inherent prepared act in the conjured-up situations. These imaginal replays are done in an atmosphere of safety and can be combined with instructions of focusing on breathing, or by adding external sensory stimulation as well. This results in a process with a combination of negative and positive affects, the original event can finally be re-experienced without fear, and the final outcome will be an emotionally less disturbing memory. When this stage is completed there is space for a new memory of the original events, and this is done as imagination of how the original event should have been in a positive fantasy. This is a repair fantasy that will be added to the memories associated with the traumatic event. This protocol with different versions of an event is close to the hypnodrama protocol of "the way it was, the way it was not, and the way it should have been" during imaginal exposure. ${ }^{65}$ Various body-mind techniques, such as hypnotherapy, psychodrama, EMDR, guided imagery, music therapy, drama therapy, dance therapy, movement therapy, art therapy, can be part of an active multimodal psychotherapy approach following the proposed manualized protocol of affective psychotherapy.

This view of the emotional memory as a scene opens up the use of the arts in the treatment. The nine muses of the Greek goddess of memory all offer the possibility to express emotional experiences through art. The use of the arts in psychotherapy can be summarized as activating the "play mode", the ability to get into a memory and/or a fantasy with emotional content, but still knowing that it has an "as if" quality. Play is suggested by Panksepp ${ }^{61}$ to be one of the core emotional action systems.

\section{Closure}

After processing the traumatic exposure so that it is linked less with negative associations but rather to positive alternatives, there is a new task at hand. This is about the future. In pathological emotional states there is often difficulty in bringing to mind and imagining a positive expectation. But expectations for the future can be seen as the memory of a fantasy, which can be practiced. The client is asked to imagine the future, for instance until the next session, and imagine that things will go well. This fantasy is then experienced, for instance as an inner movie.
Finally, there is time for the return of the positive feeling in the relaxation or safe-place experience. After that comes a short evaluation of the session, the client being in an adult, competent position of cooperation and evaluation.

In summary, with this memory reconsolidation protocol the aim is that there will be, in a setting of safety and control, without a fear reaction, the following new emotional memories: A) the sensory experience; B) the autonomous reaction; C) the motor program; D) a new alternative positive version; and $\mathrm{E})$ a positive future projection.

\section{Example I, a single case}

A boy aged 17 years comes to the clinic after referral by the school social worker. His complaints are feeling depressed and such somatic symptoms as fainting, nausea, and breathing difficulties. He has at times visited a somatic hospital where the examinations have not revealed any pathology.

\section{First visit}

On the first visit the patient complains mainly about difficulties in falling asleep, severe nightmares, and frequent day-time crying spells without having any hunch as to possible cognitive content or any memory associated with the complaints. He comes with his mother who is also present during all the sessions.

On the WHO-5 Wellbeing Index (WHO-5) he scored 48 , indicative of a slight depression. In the Mood and Feelings Questionnaire (MFQ) short version he reports a score of 10 , also indicating moderate depression.

In the treatment part of the session we focus on the situation of trying to fall asleep, and make a fantasy replay. It becomes clear that he has a fear that keeps him in a state of alarm. He practices calming and soothing techniques during the session in order to be better able to relax at bedtime. He also rehearses a future good falling asleep pattern while in a play mode.

\section{Second visit}

Three weeks later the patient returns for a new visit. Now he says that after an initial improvement his state has deteriorated. He has increased feelings of hopelessness, more severe mood swings, and increased suicidal ideation.

His score on the WHO-5 is 28 , indicating more severe depression. The score on the MFQ is 16 , indicating severe depression. He agrees to try affective psychotherapy and he starts by making a mood map. This map depicts the present interaction of emotional states as well as proposed changes to 
achieve a wished for future well-functioning mood regulation. A treatment plan of five sessions is designed.

\section{Third visit}

This is the first visit solely devoted to treatment. The patient starts with relaxation and well-being exercises, and then evokes the fear that hindered him from falling asleep. He experienced a bodily sensation of stiffness and when this is focused on he enters a memory related to negative family relations at the age of 10 . He explores those memories in his mind, following the treatment protocol, and the negative emotional arousal gradually decreases. The movement impulse being held back at the time was running, which he now can experience in fantasy. He then makes a fantasy replay of the way it should have been - having been consoled and protected. Finally he re-enters in a state of relaxation and well-being fantasy. The session is completed by an evaluation that was positive. He is surprised at the appearance of those episodic memories and of their strong emotionality.

\section{Fourth visit}

On the fourth visit 5 weeks later, after Christmas vacation, he says that he wants to stop the treatment. He came only to say thank you because now he feels well. He has no problem falling asleep, no anxiety spells, and no somatic complaints. His score in MFQ is 0 and in WHO-5 92, indicating good mental health.

\section{Fifth visit}

At a scheduled follow-up visit 3 months later he is still sleeping well, had no somatic complaints, and no anxiety spells. His score on the MFQ is 0 and on WHO-5 92. When asked why he thought his mental status had improved, he refers to the treatment given.

\section{Reflection}

The results in this single case were good and showed the possibility of adapting the proposed protocol. We must, however, caution that symptomatology is not always dissolved that easily. In this case, the presence of a safe person during the treatment sessions might have added to the treatment effect.

\section{Example 2, a case series}

In a case series of 14 suicidal adolescents it was found that they presented a post-trauma reaction with suicidality, insomnia, bodily symptoms, and disturbed mood regulation. ${ }^{66}$ They were treated according to the psychotherapy treatment protocol presented in this article. Of the adolescents 13 out of 14 had lost their severe symptoms within 4 to 20 treatment sessions. The Global Assessment of Function was judged before treatment, directly after treatment and at 22 months post-treatment. The significant change towards normality after treatment was still present at the 22-month follow-up.

\section{Summary and prospects}

This paper has attempted to extract important aspects of present knowledge in the neurocognitive science of emotions and to apply these to the treatment of disturbing reactions with negative emotional memories. We believe that this is an emerging field and that clinical practice and affective neuroscience can mutually interact to improve the treatment of suffering individuals. Based on the theory of memory reconsolidation, the approach presented focuses on changing old emotionally negative episodic memories and on creating new positive memories. It also hypothesizes that the motor component in the emotional memory is central to pathology; emotion and action preparedness being inseparable. These ideas should be further tested. The manualized approach presented, the treatment framework of affective psychotherapy, is free to be tested and developed.

\section{Disclosure}

The authors report no conflicts of interest.

\section{References}

1. Oppenheim D. Die Traumatischen Neurosen. Berlin: Hirschwald; 1892.

2. Kessler RC, Sonnega A, Bromet A, et al. Posttraumatic stress disorder in the National Comorbidity Survey. Arch Gen Psychiatry. 1995;52:1084-1060.

3. Frans Ö, Rimmö PA, Åberg L, Fredrikson M. Trauma exposure and posttraumatic stress disorder in the general population. Acta Psychiatr Scand. 2005; 11:291-299.

4. Al-Saffar S, Borgå P, Edman G, Hällström T. The aetiology of posttraumatic stress disorder in four ethnic groups in outpatient psychiatry. Soc Psychiatry Psychiatr Epidemiol. 2003;38:456-462.

5. Risch N, Herrell R, Lehner T, Liang KY, Eaves L, Hoh J. Interaction between the serotonin transporter gene (5-HTTLPR), stressful life events, and risk of depression. JAMA. 2009;23:2462-2471.

6. Darves-Bornoz JM. Rape-related psychotraumatic syndromes. Eur $J$ Obstet Gynecol Reprod Biol. 1997;71:59-65.

7. Freeman D, Fowler D. Routes to psychotic symptoms: trauma anxiety and psychosis-like experiences. Psychiatry Res. 2009;169:107-112.

8. Morrison AP, Frame L, Larkin W. Relationships between trauma and psychosis: a review and integration. $\mathrm{Br} J$ Clin Psychol. 2003;42:331-353.

9. Chen WC, Chu H, Lu RB, et al. Efficacy of progressive muscle relaxation training in reducing anxiety in patients with acute schizophrenia. J Clin Nurs. 2009;18:2187-2196.

10. Van der Hart O, Witztum E, Friedman B. From hysterical psychosis to reactive dissociative psychosis. J Trauma Stress. 1993;6:43-64.

11. Felitti VJ, Anda RF, Nordenberg D, et al. Relationship of childhood abuse and household dysfunction to many of the leading causes of death in adults. The Adverse childhood Experiences (ACE) Study. Am J Prev Med. 1984;14:245-258. 
12. Van der Kolk BA. Developmental trauma disorder. Psychiatr Ann. 2005;35:401-408.

13. D'Esposito M. Functional imaging of neurocognition. Semin Neurol. 2000;20:487-498.

14. Jovanovic T, Norrholm SD, Fernell JE, et al. Posttraumatic stress disorder may be associated with impaired fear inhibition in relation to symptom severity. Psychiatry Res. 2009;167:151-160.

15. Wheeler RE, Davidson RJ, Tomakren AJ. Frontal brain asymmetry and emotional reactivity: a biological substrate of affective style. Psychophysiology. 1993;30:82-89.

16. Osgood C, Suci G, Tannenbaum P. The Measure of Meaning. Urbana: Urbana; 1957.

17. Rauch SL, Shin LM, Dougherty DD, et al. Neural activation during sexual and competitive arousal in healthy men. Psychiatry Res. 1999;91:1-10.

18. Reiman EM, Lane RD, Ahern GL, et al. Neuroanatomical correlates of externally and internally generated emotion. Am J Psychiatry. 1997; 154:918-925.

19. Rudrauf, Lachaux JP, Damasio A, et al. Enter feelings: somatosensory responses following early stages o visual induction of emotion. Int $J$ Psychophysiol. 2009;72:12-23.

20. Sabatinelli D, Lang PJ, Bradley MM, Flaisch T. The neural basis of narrative imagery: emotion and action. Prog Brain Res. 2006;156:93-103.

21. Damasio AR. Time-locked multiregional retroactivation: A systemslevel proposal for the neural substrate of recall and cognition. Cognition. 1989;33:25-62.

22. Domjan M, Burkhard B. The Principles of Learning and Behaviour. Monterey: Brooks/Cole; 1982.

23. Wolpe J. Psychotherapy by Reciprocal Inhibition. Stanford: Stanford University Press; 1958.

24. Lang PJ. Imagery in therapy: an information processing analysis of fear. Behav Ther. 1977;8:862-886.

25. Thach WT. On the specific role of the cerebellum in motor learning and cognition: clues form PET activation and lesion studies in man. Behav Brain Sci. 1996;19:411-431.

26. Bischoff-Grethe A, Ivry RB, Grafton ST. Cerebellar involvement in response reassignment rather than attention. J Neurosci. 2002;22: 546-553.

27. Critchley HD, Corfield DR, Chandler CJ, et al. Cerebral correlates of autonomic cardiovascular arousal: a functional neuroimaging investigation in humans. J Physiol. 2000;523:259-270.

28. Lane RD, Reiman EM, Ahern GL, et al. Activity in medial prefrontal cortex correlates with vagal component of heart rate variability during emotion. Brain Cogn. 2001;47:97-100.

29. Steriade M, Llinas RR. The functional states of the thalamus and the associated neuronal interplay. Physiol rev. 1988;68:649-741.

30. Högberg G. Post-Traumatic Stress Disorder: Neurobiology and Effects of Eye Movement Desensitization and Reprocessing. Stockholm; Karolinska Institutet; 2008.

31. Luys JB. Traité Clinique et Pratique des Maladies Mentales. Paris: Delahaey el Lecrosnier; 1881.

32. Pagani M, Högberg G, Salmaso D. et al. Regional cerebral blood flow during auditory recall in 47 subjects exposed to assaultive and nonassaultive trauma and developing or not posttraumatic stress disorder. Eur Arch Psychiatry Clin Neurosci. 2005;255:359-365.

33. Ketter TA, Andreason PJ, George MS. Anterior paralimbic mediation of procaine-induced emotional and psychosensory experiences. Arch Gen Psychiatry. 1996;53:59-69.

34. Metzger LJ, Carson MA, Paulus LA, Paige SR, Lasko NB, Pitman RK. PTSD arousal and depression symptoms associated with increased right-sided parietal EEG asymmetry. J Abnorm Psychol. 2004;113(2):324-329.

35. Achuff P. The lateralization of emotion. Brain and Mind Magazine. 2001;14.

36. Alves NT, Aznar-Casanova JA, Fukusima SS. Patterns of brain asymmetry in the perception of positive and negative facial expressions. Laterality. 2009;14:256-272.
37. Schiffer F, Teicher MH, Anderson C, et al. Determination of hemispheric emotional valence in individual subjects: a new approach with research and therapeutic implications. Behav Brain Funct. [serial on the internet] 2007;3(13).

38. Thayer JF, Brosschot JF. Psychosomatics and psychopathology: looking up and down from the brain. Psychoneuroendocrinology. 2005;30:1050-1058.

39. Heinzel A, Bermpohl F, Niese R, et al. How do we modulate our emotions? Parametric fMRI reveals cortical midline structures as regions specifically involved in the processing of emotional valences. Brain Res Cogn Brain Res. 2005;25:348-358.

40. Büchel C, Morris J, Dolan R, Friston K. Brain systems mediating aversive conditioning: an event-related fMRI study. Neuron. 1998;20:947-957.

41. Kosten TR, Mason JW, Giller EL, Ostroff RB, Harkness L. Sustained urinary norepinephrine and epinephrine elevation in post-traumatic stress disorder. Psychoneuroendocrinology. 1987;12:13-20.

42. Breslau N. Neurobiological research on sleep and stress hormones in epidemiological samples. Ann N Y Acad Sci. 2006;1071:221-230.

43. Mellman TA, Kumar A, Kulick-Bell R, Kumar M, Nolan B. Nocturnal/ daytime urine noradrenergic measures and sleep in combat-related PTSD. Biol Psychiatry. 1995;38:174-179.

44. Murburg MM, McFall ME, Lewis N, Veith RC. Plasma norepinephrine kinetics in patients with posttraumatic stress disorder. Biol Psychiatry. 1995;38:819-825.

45. O'Donnell T, Hegadoren KM, Coupland NC. Noradrenergic mechanisms in the pathophysiology of post-traumatic stress disorder. Biol Psychiatry. 2004;50:273-283.

46. Jovanovic T, Norrholm SD, blanding NQ, et al. Impaired fear inhibition is a biomarker of PTSD but not depression. Depress Anxiety. 2010;27:244-251.

47. Yui K, Goto K, Ikemoto S, Ishiguro T. Monoamine neurotransmitter metabolites and spontaneous recurrence of methamphetamine psychosis. Brain Res Bull. 1997;43:25-33.

48. Osuch EA, Benson B, Geraci M, et al. Regional cerebral blood flow correlated with flashback intensity in patients with posttraumatic stress. Biol Psychiatry. 2001;50:246-253.

49. Liberzon I, Taylor SF, Fig LM, Koeppe RA. Alterations of corticothalamic perfusion ratios during a PTSD flashback. Depress Anxiety. 1996/1997;4:146-150.

50. Huber M, Siol T, Herholz K, Lenz O, Köhle K, Heiss WD. Activation of thalamo-cortical systems in post-traumatic flashbacks: a positron emission tomography study. Traumatology. 2001;7:131-141.

51. Linden DEJ. How psychotherapy changes the brain - the contribution of functional neuroimaging. Mol Psychiatry. 2006;11:528-538.

52. Pagani M, Gögberg G, Dario Salmaso, et al. Effects of EMDR psychotherapy on ${ }^{99 \mathrm{tm} T c-H M P A O}$ distribution in occupationrelated post-traumatic stress disorder. Nucl Med Commun. 2007;28: 757-765.

53. Bryant RA, Felmingham K, Kemp A, et al. Amygdala and ventral anterior cingulated activation predicts treatment response to cognitive behaviour therapy for post-traumatic stress disorder. Psychol Med. 2008;38:555-561.

54. Nader K, Schafe GE, Le Doux JE. Fear memories require protein synthesis in the amygdala for reconsolidation after retrieval. Nature. 2000;406:722-726.

55. Forcato C, Burgos VL, Argibay PF, et al. Reconsolidation of declarative memory in humans. Learn Mem. 2007;14:295-303.

56. Nadel L, Moscovitch M. Memory consolidation, retrograde amnesia and the hippocampal complex. Curr Opin Neurobiol. 1997;7: $217-227$.

57. Nadel L, Campbell J, Ryan L. Autobiographical memory retrieval and hippocampal activation as a function of repetition and the passage of time. Neural Plast. 2007; 90472.

58. Monfils MH, Cowansage KK, Klann E, LeDoux JE. Extinctionreconsolidation boundaries: key to persistent attenuation of fear memories. Science. 2009;324(5929):951-955. 
59. Forcato C, Rodriguez MLC, Pedreira ME, Maldonado H. Reconsolidation in humans opens up declarative memory to the entrance of new information. Neurobiol Learn Mem. 2010;93(1):77-84.

60. Delgado MR, Jopu RL, Ledoux JE, et al. Avoiding negative outcomes: tracking the mechanisms of avoidance learning in humans during fear conditioning. Front Behav Neurosci. 2009;3:1-9.

61. Panksepp J. Affective consciousness: core emotional feelings in animals and humans. Conscious Cogn. 2005;14:30-80.

62. Nardo D, Högberg G, Flumeri F, et al. Selfrating scales assessing subjective well-being and distress correlate with rCBF in PTSD-sensitive regions. Psychol Med. 2011. [Epub ahead of print].
63. Duncan BL, Hubble MA, Miller SD. The Heart and Soul of Change: What Works in Therapy. Washington: American Psychological Association; 2010.

64. Elman D. Hypnotherapy. Glendale: Westwood Publishing Co; 1964.

65. Högberg G. International Association of Group Psychotherapy Abstracts. Buenos Aires: IAGP; 1995.

66. Högberg G, Hällström T. Active multimodal psychotherapy in children and adolescents with suicidality: description, evaluation and clinical profile. Clin Child Psychol Psychiatry. 2008;13:435-448.

\section{Publish your work in this journal}

Psychology Research and Behavior Management is an international, peerreviewed, open access journal focusing on the science of psychology and its application in behavior management to develop improved outcomes in the clinical, educational, sports and business arenas. Specific topics covered include: Neuroscience, memory \& decision making; Behavior modification \& management; Clinical applications; Business \& sports performance management; Social and developmental studies; Animal studies. The manuscript management system is completely online and includes a quick and fair peer-review system. Visit http://www.dovepress. com/testimonials.php to read real quotes from published authors.

Submit your manuscript here: http://www.dovepress.com/psychology-research-and-behavior-management-journal 